\title{
TRANSPORTE DE MERCANCÍAS POR FERROCARRIL EN ESPAÑA; AGOTAMIENTO DE UN MODELO Y SU NECESARIA RENOVACIÓN. EL DIFÍCIL FUTURO
}

\author{
José María Serrano Martínez \\ Departamento de Geografía. Universidad de Murcia \\ jmserran@um.es
}

\section{RESUMEN}

El transporte de mercancías por ferrocarril en España se ha reducido con intensidad durante los últimos años. En el presente significa proporciones mínimas, casi amenaza su desaparición. Las causas que han motivado ese proceso son plurales y de diferente naturaleza. Atendiendo a varios criterios, económicos, espaciales, estratégicos, etc. se aconseja su recuperación. Pero, eso conlleva numerosas dificultades. El plan estratégico presentado por el Gobierno busca su relanzamiento. Se analiza su contenido y sus debilidades. A la vez, se presentan algunas alternativas y consideraciones para conseguir incrementar su capacidad de tráfico.

Palabras clave: ferrocarril, transporte de mercancías, modelo de transporte.

\section{ABSTRACT}

The transport of goods for railway in Spain has decreased with intensity during the last years. Presently it means minimum proportions, almost threat their disappearance. The causes that have motivated that process are plural and of different nature. Assisting to several approaches, economic, spatial, strategic, etc. he seeks advice their recovery. But, that bears numerous difficulties. The strategic plan presented by the Government looks for its recovery.

Fecha de recepción: febrero 2011.

Fecha de aceptación: julio 2012. 
It is analyzed their content and their weaknesses. At the same time, they show up some alternatives and considerations to be able to increase their traffic capacity.

Key words: railway, transport of goods, model of transport.

\section{INTRODUCCIÓN Y PLANTEAMIENTO}

Los transportes, en sus diferentes modos, constituyen elementos esenciales en la vertebración del territorio. La intensidad y dirección de los flujos de tráfico son componentes importantes en la configuración espacial. Están vinculados a la disposición de la red urbana y a la propia organización de las actividades económicas, en su producción, destino o consumo. Dada su trascendencia, la organización conjunta de los transportes, el denominado sistema de transportes, precisa un planeamiento completo y minucioso para su adecuado funcionamiento. Éste, debe ensamblar de manera armónica las diferentes escalas territoriales. Desde la local a la más amplia. No hay lugar para aislamientos ni particularismos. En el mundo presente, crecientemente integrado, los desajustes en las escalas pueden llevar a situaciones de desenclave de la que se derivan tremendos costes. Los territorios que quedan fuera sufren pronto las consecuencias (Quinet, 1992). Las dificultades de accesibilidad van unidas a reducciones en la competitividad, y viceversa (Perenne, 1995). La mayor duración de tiempo invertido en los transportes, de personas y mercancías, así como sus costes más elevados, significan mermas en la concurrencia, dificultades para futuras inversiones. A partir de eso, la espiral de obstáculos no hará más que crecer. De ahí la necesidad de lograr redes de transportes, bien integradas y complementadas, que permitan un sistema fluido y global de desplazamientos, con eficiente operatividad y reducidos costes económicos y ambientales.

Durante años, todo el sistema productivo mundial se ha comportado siguiendo un esquema que marcaba un horizonte continuo de crecimiento, con uso ilimitado de los recursos. Ahora, por el contrario, dentro de una concepción sistémica global, todo lo relacionado con la sostenibilidad, en su proyección a largo plazo, introduce un nuevo escenario. La dimensión energética, su valor y su carga ecológica negativa, alcanza renovada atención. Es necesario favorecer su ahorro y minimizar sus impactos desfavorables.

El asunto central analizado aquí es el transporte de mercancías a través del modo ferroviario en España. El problema radica en su reducido volumen y en su evolución decreciente; tanto en datos absolutos como relativos representa valores muy escasos. Apenas cuenta en el conjunto del tráfico nacional. En el transcurso de los últimos años ha registrado una disminución progresiva (López, 1987). Aunque se plantean ciertos proyectos de mudanza, nada parece apuntar que sea factible un cambio de tendencia, ni menos aún, una modificación de la estructura modal existente. Sin embargo, en teoría, hay numerosos aspectos que confirman las ventajas de conseguir un uso mayor. La estrategia nacional e internacional aconseja su revitalización. Pero eso precisa un planeamiento conjunto de gran alcance, lo cual no resulta sencillo emprender; menos aún en una situación generalizada de crisis económica como la actual. No se presume sencillo encontrar financiación para las considerables inversiones que precisa esa nueva meta, en sus diferentes apartados.

Los objetivos marcados en este artículo se focalizan en los siguientes aspectos: 
- Análisis de la realidad presente, dentro del contexto global del tráfico de mercancías.

- Estudio de las causas esenciales que han llevado a su escasa utilización.

- Delimitar las principales consecuencias derivadas para el movimiento de mercancías y el conjunto del sistema productivo.

- Presentar algunas opciones y sugerencias de cambio y revitalización.

El marco espacial de análisis abarca la escala nacional, como ámbito básico; pero insertada en otra más la Europa Comunitaria (Bauchet, 1996); todo lo cual representa y precisa interpretarlo dentro de una geoestrategia superior.

Para abordar esas cuestiones es necesario utilizar una metodología mixta. Se realiza un conciso estudio de su evolución temporal reciente, previa a la situación actual. Ésta se desmenuza mediante una tarea de análisis, centrada en las principales cuestiones implicadas; eso se completa con procedimientos de síntesis; único camino que permite alcanzar una visión global de la compleja realidad existente.

\section{REDUCCIÓN PROGRESIVA DEL TRANSPORTE FERROVIARIO DE MERCANCÍAS. SUS CAU- SAS Y SITUACIÓN ACTUAL}

Los diferentes datos que miden la evolución del transporte de mercancías en España, referidos a las últimas décadas, confirman una reducción progresiva y continuada. En cierta forma eso se produce de manera general en la mayoría de los países del mundo (Faith, 1990). Durante el siglo XIX, tras el inicio del ferrocarril, se emprende un auge ferroviario generalizado; por el contrario en el XX, en especial durante su segunda mitad, se advierte un ascenso de la carretera, en parte a costa de este modo (Izquierdo, 1992). Numerosas causas, a las que se hará referencia más adelante, contribuyen a ello.

Lo peculiar del caso español radica en la acusada merma que registran los ferrocarriles. En especial ésta resulta abultada dentro de un periodo caracterizado, en general, por el continuo crecimiento económico y el aumento consiguiente de los flujos de transportes. Basta señalar que la base 100 del total de transporte interior de mercancías de 1970, se convierte en 213,32 en 2010. También el transporte de mercancías de ámbito internacional aumenta con brío. En ese mismo periodo temporal pasa de la base 100 de 1970 a 239,41. En ambos ámbitos, pues, se duplica la cantidad inicial generosamente. Tal aumento espectacular se debe, de una parte, al propio incremento de la actividad productiva. De otra, a una palpable desorganización y descoordinación del modelo de transporte y producción, en tanto que aquel debe ser el medio de relacionar estas áreas con las de consumo, en sus diferentes escalas y ámbitos. Eso explica que el total de mercancías transportadas en España representa una cantidad superior a la de Francia (con un PIB casi el doble del español), y cercano al doble de Italia (cuyo PIB, también es mayor al nuestro). El movimiento de mercancías en España supone casi dos veces los índices habituales, registrados por la media europea (VV. AA., 2009). De esa manera, y circunscribiéndonos al marco nacional, numerosos productos fabricados en el norte, se venden en el sur; y otros semejantes que se producen en el este, se consumen en el oeste. Todo eso debe llevarnos a una reflexión sobre la ordenación del territorio y la dispar distribución de activos de transformación y consumo. Es conveniente reducir la movilidad innecesaria de mercancías. El coste económico creciente que ello implica y su impacto medio ambiental así lo aconsejan. 
Sin embargo, en medio de esa dinámica de incremento del traslado de mercancías, las transportadas por ferrocarril descienden de manera paulatina y casi constante. Los datos escuetos se especifican en el cuadro 1.

Cuadro 1

MERCANCÍAS TRANSPORTADAS POR FERROCARRIL EN ESPAÑA

\begin{tabular}{|c|c|c|c|c|c|c|c|c|}
\hline & \multicolumn{2}{|c|}{ Total } & \multicolumn{2}{c|}{ RENFE-Operadora } & \multicolumn{2}{c|}{ FEVE } & \multicolumn{2}{c|}{$\begin{array}{c}\text { Comp. CC.AA., } \\
\text { Particulares }\end{array}$} \\
\hline & $\begin{array}{c}\text { Millones } \\
\text { TM }\end{array}$ & TM/Km. & $\begin{array}{c}\text { Millones } \\
\text { TM }\end{array}$ & TM/Km. & $\begin{array}{c}\text { Millones } \\
\text { TM }\end{array}$ & TM/Km. & $\begin{array}{c}\text { Millones } \\
\text { TM }\end{array}$ & TM/Km. \\
\hline 1970 & 43,2 & 10.339 & 38,9 & 9.693 & 0.2 & 12 & 12,1 & 634 \\
\hline 1980 & 46,9 & 11.282 & 36,5 & 10.888 & 4,7 & 171 & 5,7 & 223 \\
\hline 1985 & 41,1 & 12.074 & 31,7 & 11.653 & 5,0 & 183 & 4,4 & 228 \\
\hline 1990 & 37.3 & 11.613 & 28,1 & 11.206 & 4,3 & 205 & 3,9 & 202 \\
\hline 1995 & 30,0 & 10.419 & 25,1 & 10.077 & 3,0 & 211 & 2,8 & 131 \\
\hline 2000 & 30,7 & 12.171 & 25,8 & 11.620 & 3,2 & 451 & 1,7 & 100 \\
\hline 2005 & 31,1 & 11.641 & 25,5 & 11.071 & 3,5 & 465 & 2,1 & 105 \\
\hline 2006 & 31,3 & 11.599 & 24,9 & 11.011 & 4,1 & 473 & 2,3 & 115 \\
\hline 2007 & 30,9 & 11.124 & 14,7 & 10.547 & 3,9 & 460 & 2,3 & 117 \\
\hline 2008 & 27,4 & 10.287 & 22,0 & 9.937 & 3,0 & 429 & 2,4 & 121 \\
\hline 2009 & 21,0 & 9.391 & 16,6 & 6.973 & 2,8 & 339 & 1,6 & 79 \\
\hline
\end{tabular}

Fuente: Ministerio de Fomento.

Los diferentes segmentos indicados, con algunos matices que amortiguan su ritmo, confirman la disminución progresiva. Este apartado parece haber entrado en una espiral de declive, con amenaza de su propia continuidad; casi anuncian su colapso futuro, como modo de transporte propio, frente a otras alternativas de desplazamiento.

Al comparar el aumento constante del transporte en España, con el descenso progresivo del modo ferroviario, se constata su reducción continuada. Así, en el tráfico interior ha pasado de contabilizar una tasa del 8,89\% en 1975 a menos del $3 \%$ en 2010. También en el ámbito internacional su debilitamiento es palpable. En esas mismas fechas se encoge desde el modesto 1,70 \% inicial al 1,26\%. Tales proporciones casi pueden calificarse de marginales. Son mucho más reducidas a las de otros países vecinos, donde aún siendo modestas, superan ampliamente las cuotas españolas (VV. AA., 2009). Si nos fijamos con detalle en la naturaleza, estructura y composición del transporte de mercancías, los datos aclaran sus características y explican sus debilidades; se especifican en el cuadro 2. 
Cuadro 2

EVOLUCIÓN DE LA ESTRUCTURA DEL TRANSPORTE DE MERCANCÍAS POR FERROCARRIL

\begin{tabular}{|c|c|c|c|c|c|c|c|}
\hline & $\mathbf{1}$ & $\mathbf{2}$ & $\mathbf{3}$ & $\mathbf{4}$ & $\mathbf{5}$ & $\mathbf{6}$ & $\mathbf{7}$ \\
\hline 1970 & 43,2 & $71,52 \%$ & -- & -- & -- & -- & - \\
\hline 1975 & 46,8 & 80,55 & $86,27 \%$ & $1,57 \%$ & $1,20 \%$ & $2,33 \%$ & 8,52 \\
\hline 1980 & 46,9 & 77,82 & 82,78 & 4,06 & 1,54 & 2,30 & 9,38 \\
\hline 1985 & 41,1 & 77,12 & 79,78 & 9,53 & 1,47 & 2,31 & 6,82 \\
\hline 1990 & 37,3 & 78,01 & 74,16 & 13,28 & 1,13 & 2,36 & 8,91 \\
\hline 1995 & 30,0 & 80,66 & 78,15 & 18,31 & 1,64 & -- & 2,02 \\
\hline 2000 & 30,7 & 84,03 & 68,66 & 29,44 & -- & -- & 1,90 \\
\hline 2005 & 31,1 & 81,99 & 70,36 & 27,21 & -- & -- & 2,43 \\
\hline 2009 & 21.1 & 79,04 & 72,47 & 27,30 & -- & -- & 0,23 \\
\hline
\end{tabular}

1: Total Millones TM.; 2: Porcentaje correspondiente a RENFE; 3: Vagón completo; 4: Contenedores; 5: Detalle y paquete exprés; 6: Correos; 7: Servicios.

Fuente: Elaboración propia sobre datos del Ministerio de Fomento.

La destacada significación de RENFE es una constante. Tanto cuando ejercía su monopolio estatal, como tras la liberalización (2005) y su funcionamiento como empresa operadora. Esto no debe extrañar; en gran medida, es reflejo de comportamientos comunes en el ámbito Europeo Occidental. De forma singular se advierte que la denominada «modalidad del vagón completo» sobresale sobre otros usos. Al tiempo se indica un ascenso de la significación del tráfico de contenedores. Aunque en la última década confirma un estancamiento. Este apartado constata la debilidad del tráfico ferroviario en España; pues el tremendo auge y la generalización progresiva del empleo de contenedores (uno de los rasgos del incremento del transporte actual de mercancías), no han encontrado en el ferrocarril en España una vía adecuada. Se ha desperdiciado la ocasión de relanzar y afianzar tal medio operativo. Ahí radica una de sus mayores debilidades presentes; su recuperación constituye un reto a superar en el futuro.

Tras esos datos escuetos que muestran una realidad palpable, interesa buscar las causas básicas que han llevado a ello. Una explicación sucinta se aporta a continuación. No conviene olvidar una serie de circunstancias objetivas que limitan este modo de transporte. La configuración peninsular española conlleva un cierto aislamiento natural hacia los países vecinos; ello limita el desarrollo del ferrocarril. La propia orografía accidentada y su disposición interior tampoco favorecieron, desde el principio, el trazado inicial de los ramales ferroviarios. Estos quedaron condicionados, a su vez, por la morfología de la malla básica de ciudades. Esos elementos iniciales se han mantenido, en gran medida, hasta hoy, con ligeras correcciones. De esa forma, la red de ferrocarriles, en su trazado inicial, adquirió una morfología que, como se ha apuntado, continúa en gran medida (Wais, 1974). La elección del ancho de vía ibérico, aún dominante, tampoco ha ayudado a ello (Izquierdo, 1993).

No obstante, las principales causas que explican la situación actual encuentran su razón principal en las decisiones emprendidas en tiempos más recientes. En especial, durante los 
últimos treinta años. Todo eso ha llevado a que, en el apartado concreto del transporte de mercancías por ferrocarril, las carencias tradicionales, de infraestructuras y otros apartados, apenas se han modificado. Desde una perspectiva absoluta, las mejoras han sido mínimas. En esencia, sus causas obedecen a una doble naturaleza, estratégica y económica; si bien, ambas se interrelacionan, participan y se combinan en su devenir. Unas breves consideraciones se añaden al respecto.

Estratégicas. No se ha confeccionado un plan de transportes continuado que abarque y se mantenga durante tiempo, de manera efectiva. Incluso, algunos de los más recientes y ambiciosos programas, Plan Director de Infraestructuras, 1993-2007 (MOPT, 1993) y Plan Estratégico de Infraestructuras y Transportes (acrónimo, PEIT), (2004), - este último con un horizonte de actuaciones que abarca de 2005 a 2020 - , ambos contienen enfoques y prioridades diferentes, casi opuestos, en algunos apartados. Cuando se analiza su devenir, desde la situación actual, (inicios de 2011), se advierte que, en gran medida, ha primado durante más tiempo la opción de la carretera. La atención dedicada al ferrocarril, sólo creciente en años más próximos, se ha hecho pensando, sobre todo, en el tráfico de pasajeros. No en las mercancías. Éste ha quedado pospuesto, casi en una situación marginal.

Para entender y explicar lo sucedido, sin intentar justificarlo, hay que situarse en el escenario de España cuando inicia su transición democrática y al poco comienzan las grandes inversiones en infraestructuras de transporte. Tras años de fuerte crecimiento económico y de aumento del nivel de vida, la demanda de tales infraestructuras era acuciante. A la vez, la débil oferta existente de ellas, amenazaba con estrangular y limitar el dinamismo de una economía que pugnaba por incorporarse a la Comunidad Económica Europea. Pero, aún se vivía bajo el deslumbramiento general de considerar a la carretera como medio operativo plural y de mayor utilidad. Esas inversiones prometían satisfacción y eco social, inmediato y favorable, algo poco común. La ductilidad que ofrece la carretera, con su uso mixto, pasajeros y mercancías, junto a la mayor permeabilidad de su penetración espacial y la menor ruptura de carga, ayudaban a sobrevalorarla, frente a consideraciones menos adecuadas de los ferrocarriles.

Así mismo, en España, la rápida consolidación del nuevo modelo autonómico, introducía otro sesgo territorial a tener en cuenta. A menudo, esa realidad ha dificultado emprender una estrategia de ámbito nacional español; otro elemento inherente a la política ferroviaria.

Económicas. Las disponibilidades económicas casi siempre suelen ser exiguas, frente a las necesidades de gasto. También, la escasa continuidad de las inversiones añade incertidumbre. Esto lógicamente puede justificar la toma de muchas decisiones coyunturales, que luego resultan poco acertadas. También, como ya se ha apuntado, las carencias en infraestructuras de transportes en España han sido tradicionalmente tan elevadas, que aconsejaba marcar prioridades. Con todo ello no se desean justificar las decisiones tomadas, al primar la carretera. Sólo se intenta entender, en su justa dimensión, el contexto global que permita interpretar con mayor justeza lo acaecido (García, 1987). Los datos del cuadro 3 permiten calibrar la dimensión de esos hechos. 
Cuadro 3

INVERSIÓN EN INFRAESTRUCTURAS EN ESPAÑA (\%)

\begin{tabular}{|l|c|c|c|c|c|c|c|}
\hline & Viarias & Ferrocarriles & Aeropuertos & Portuarias & Hidráulicas & Urbanas & Otras \\
\hline $1981-85$ & 21,8 & 8.4 & 2,0 & 3,0 & 15,8 & 6,1 & 42,7 \\
\hline $1986-90$ & 28,6 & 8,9 & 1,8 & 2,8 & 28,6 & 9,4 & 34,9 \\
\hline $1991-95$ & 36,0 & 8,1 & 1,9 & 3,0 & 36,0 & 7,7 & 30,3 \\
\hline $1996-00$ & 30,8 & 10,1 & 2,8 & 2,7 & 30,8 & 8,5 & 32,3 \\
\hline $2000-05$ & 23,7 & 13,7 & 5,0 & 2,6 & 23,7 & 6,8 & 39,3 \\
\hline
\end{tabular}

Fuente: Elaboración propia, datos del I. V. I. E.

Durante el último cuarto de siglo las infraestructuras viarias por carretera, en su sentido genérico, ha sido un apartado favorecido. Así se ha alcanzado una significativa mejora en la oferta de la red viaria. Destaca sobre manera la puesta en servicio de $13.000 \mathrm{kms}$. de nuevas vías rápidas de gran capacidad. Se ha pasado de contar con unos escasos trechos, a una malla tupida, de las más completas del mundo (Serrano 2008). El balance, en ese sentido, es bastante satisfactorio. Más discutible resulta, a largo plazo y desde una perspectiva estratégica, la relegación que tal opción ha supuesto para el ferrocarril; con las consecuencias que de ello se derivan para los diferentes aspectos relacionados con los transportes.

Conviene fijarse con mayor detalle en la auténtica dimensión económica de la actividad inversora realizada. Para abreviar el texto, el cuadro 4, detalla la cantidad de esos valores. Se especifican sólo los datos de los sucesivos lustros, para tomarlos como referencia de su magnitud y proporción variable al paso de los mismos.

\section{Cuadro 4}

INVERSIONES REALES DEL MINISTERIO DE FOMENTO (ANTES M.O.P.U. Y M.O.P.T.), ORGANISMOS AUTÓNOMOS Y SOCIEDADES ESTATALES RECONOCIDAS

\begin{tabular}{|c|c|c|c|c|}
\hline & \multicolumn{2}{|c|}{ Ferrocarriles } & \multicolumn{2}{c|}{ Carreteras } \\
\hline & Millones euros* & \% total & Millones euros & \% total \\
\hline 1981 & 86,33 & 20,61 & 147.86 & 35,30 \\
\hline 1985 & 228,15 & 22,41 & 404,64 & 39,76 \\
\hline 1990 & 383,62 & 10,77 & 2.131 .84 & 59,88 \\
\hline 1995 & 378,06 & 13,89 & $2.259,74$ & 83,08 \\
\hline 2000 & 354.43 & 13.07 & $2.283,16$ & 64,12 \\
\hline
\end{tabular}

Fuente: Elaboración propia, sobre datos de los anuarios ministeriales correspondientes *Valores calculados en precios corrientes.

Se advierte el aumento continuo de las cifras y los montantes alcanzados. Interesa recordar que, en buena medida, tal aumento espectacular ha sido posible gracias a las ayudas de financiación procedentes de la Unión Europea (González \& Benedicto, 2006). Es imposible imaginar sin su concurso la dimensión de las inversiones realizadas. 
La mejora y ampliación de la red viaria española, además de favorecer la vertebración nacional, ha espoleado la demanda interna, trasladando sus efectos indirectos e inducidos a numerosos apartados de la vida productiva. No es fácil entender la dimensión de algunas destacadas empresas españolas relacionadas con ese segmento de la actividad económica, sin ese incremento inusitado de las infraestructuras (Fernández, 2006). Se tuvo la habilidad de que la fuerte demanda de actividades relacionadas con su construcción de tantas infraestructuras fuera cubierta por empresas nacionales. El reto que ello representó espoleó la actividad empresarial. Ahora, (2011), con otro escenario bien diferente, esas empresas tienen que buscar su trabajo en latitudes alejadas. Todo eso debe invitar a la reflexión y al análisis más completo de la complejidad de los hechos, para no sacar conclusiones apresuradas.

Además, también ha influido en el vivo incremento del parque general de vehículos y de los flujos de tráfico. Así, el parque móvil pasa de 10,19 millones de unidades totales en 1980 a más de 31 millones en 2010. En lo que concierne sólo a los vehículos dedicados al transporte de mercancías, su incremento también ha sido sustancial, asciende de 1,33 millones en 1980 a 5,13 en 2009. Ambas cifras explican el auge de este modo de transporte, con la consiguiente reducción de otros, ya comentada. Por su parte, la opción tomada, por el sistema de autovías de uso gratuito, ha contribuido a aumentar la demanda; se ha impulsado el uso de la circulación por carretera. Los costes inmediatos de uso han estado subvencionados de manera directa por las inversiones públicas. Con el auge de ese modelo de transporte se han consumido enormes recursos. Ahora bien, este modelo constituía una oferta popular y gratificante. Quienes lo auspiciaban se sentían recompensados, obtenían ventajas inmediatas con ello. Más aún, su efecto multiplicador se ha trasladado a otros sectores productivos. En consecuencia, eso ha sido uno de los pilares impulsores del crecimiento económico español durante lustros.

Pero, la opción elegida del protagonismo de la carretera ahora se advierte que se sustentaba en una estrategia de cortas miras. Pasados unos años se constatan enormes disfunciones en el sistema de transportes articulado. Son apreciables sus carencias, en especial, en todo lo concerniente al tráfico de mercancías. Además de sus significativas consecuencias relacionadas con el mantenimiento, los costes medio ambientales y su difícil sostenibilidad. Sus efectos, pues, en el orden económico, son de considerable envergadura. Así mismo, debido a su enorme trascendencia dentro del sistema productivo sus efectos se trasladan también a otros diferentes apartados de la ordenación del territorio, como sucede con los vastos y complejos espacios urbanizados existentes, sus necesidades implícitas de gran movilidad y sus modos de funcionamiento cotidiano.

Sólo en los últimos años se aprecian significativos cambios en la orientación de la política de transportes y, en consecuencia, en el destino de las inversiones, cara a modificar la nueva estructura modal de los transportes. El cuadro 5 aporta algunos datos precisos al respecto, que permiten confirmar tales afirmaciones. 
Transporte de mercancías por ferrocarril en España; agotamiento de un modelo y su necesaria renovación. El difícil futuro

Cuadro 5

INVERSIÓN EN INFRAESTRUCTURAS DE TRANSPORTE EN ESPAÑA, MILLONES DE EUROS Y VALORES PORCENTUALES. DIFERENCIACIÓN MODAL

\begin{tabular}{|l|l|l|l|l|l|l|l|l|}
\hline & \multicolumn{2}{|c}{ Carreteras } & \multicolumn{2}{c|}{ Ferrocarriles } & \multicolumn{2}{c|}{ Aeropuertos } & \multicolumn{2}{c|}{ Portuarias } \\
\hline 2000 & 2.498 & 42,91 & 1.732 & 33,21 & 570 & 10,93 & 415 & 7,95 \\
\hline 2001 & 2.481 & 37,66 & 2.573 & 39,04 & 1.020 & 15,47 & 516 & 7,83 \\
\hline 2002 & 2.695 & 27,11 & 3.490 & 48,85 & 1.413 & 17.35 & 545 & 6,69 \\
\hline 2003 & 2.821 & 30,39 & 3.558 & 38,29 & 2.205 & 23.73 & 706 & 7,59 \\
\hline 2004 & 2.876 & 29,13 & 4.136 & 41.87 & 2.075 & 21.00 & 791 & 8,00 \\
\hline 2005 & 3.251 & 29,51 & 5.453 & 49.46 & 1.406 & 12,75 & 913 & 8,28 \\
\hline 2006 & 3.480 & 29,62 & 5.518 & 46,95 & 1.614 & 13,73 & 1.140 & 9,70 \\
\hline 2007 & 3.982 & 28,77 & 6.660 & 48,07 & 2.073 & 14,96 & 1.137 & 8,20 \\
\hline 2008 & 4.242 & 27,17 & 7.783 & 49,82 & 2.284 & 14,62 & 1.312 & 8,39 \\
\hline 2009 & 5.674 & 30,30 & 9.599 & 51,57 & 1.775 & 9,20 & 1.672 & 8,93 \\
\hline 2010 & 5.725 & 29,67 & 10.191 & 52,83 & 1.868 & 9,68 & 1.509 & 7,82 \\
\hline
\end{tabular}

Fuente: Elaboración propia, datos del Ministerio de Fomento.

Lo más destacado y sustancial, según se ha apuntado ya, es el ascenso del ferrocarril como destino de la principal suma de inversiones. Tras eso se encierra el objetivo de mejorar su papel dentro del sistema de transportes. Incluso, en el discurrir de esa década, asciende la inversión; pasa de significar un tercio del total a más de la mitad. La carretera, por el contrario, reduce su participación desde el 42,91\% en 2000 a menos del tercio en 2010. Los restantes modos de transporte mantienen proporciones más regulares. Queda, pues, clara, la apuesta prioritaria por el ferrocarril desde las inversiones públicas. Este acusado cambio de tendencia ya se reflejaba en el Plan Estratégico de Infraestructuras y Transportes. Un escueto cuadro de datos detalla la significación absoluta y porcentual de las principales partidas contempladas en el mismo.

Cuadro 6

ESTIMACIÓN ECONÓMICA DE LAS ACTUACIONES DEL P.E.I.T. (2005-2020)

\begin{tabular}{|l|r|r|}
\hline & Millones de euros & \multicolumn{1}{|c|}{$\%$} \\
\hline Transporte por ferrocarril, excepto actuaciones urbanas & 108.706 & 43,70 \\
\hline Transporte por carretera, excepto actuaciones urbanas & 62.785 & 25,23 \\
\hline Transporte aéreo & 15.700 & 6,31 \\
\hline Transporte marítimo y puertos & 23.460 & 9,43 \\
\hline Transporte intermodal de mercancías y viajeros & 3.620 & 1,45 \\
\hline Transporte urbano y metropolitano & 32.527 & 13,07 \\
\hline Investigación, desarrollo e innovación & 2.040 & 0,82 \\
\hline Total actuaciones previstas & 248.892 & 100,00 \\
\hline
\end{tabular}

Fuente: Ministerio de Fomento. 
Esa orientación de las inversiones públicas era novedosa y llamativa. Permite interpretarla de la siguiente forma: cubiertas, en gran medida, las necesidades de las vías rápidas de gran capacidad (VRGC), con su morfología radial y, complementada, en parte, con ciertos trechos que anuncian una red mallada; ahora la prioridad del transporte terrestre pasa a ser el ferrocarril. En concreto, las llamadas líneas de alta velocidad (acrónimo LAV), orientadas hacia el tráfico de pasajeros (Serrano \& Marín, 2010). Eso explica que el contrastado destino final de las inversiones a realizar. Una modulación opuesta a la anterior.

El tema del desarrollo y evolución de la alta velocidad ferroviaria es importante y de sobra conocido (Nijkamp \& Greenhuizen, 1997). No es este, ni el momento, ni el lugar para tratarlo. Poco se podría añadir a ello. Basta recordar que tras la puesta en servicio de su primer tramo en España en 1992, con posterioridad se han ido abriendo otros (el más reciente en Diciembre de 2010, que une las ciudades de Madrid, Cuenca, Valencia y Albacete). Al finalizar 2010 el total de Km. de LAV disponibles asciende a más de dos mil kilómetros. De manera algo triunfalista se proclama el liderazgo que ello significa dentro del marco europeo y su destacado encumbramiento mundial. Por sí mismo este auge constituye un apartado interesante, pero ajeno al objetivo central aquí estudiado. Eso no impide enfatizar su destacada trascendencia por cuanto significa un modo útil para mejorar la accesibilidad y la cohesión territorial española (Gutiérrez Puebla, et al., 1992 y 2006). Basta pensar lo que representan las numerosas capitales de provincia ya conectadas mediante LAV y su significación demográfica, económica y estratégica dentro de España.

Ahora bien, interesa remarcar un aspecto esencial, ya apuntado. Las LAV están orientadas, hasta ahora, exclusivamente al tráfico de pasajeros. El tráfico de mercancías queda al margen

Cuadro 7

EVOLUCIÓN DE LARED FERROVIARIA ESPAÑOLA

\begin{tabular}{|l|r|r|r|r|r|r|r|r|}
\hline & \multicolumn{1}{|c|}{$\mathbf{1}$} & \multicolumn{1}{|c|}{$\mathbf{1}$} & \multicolumn{1}{|c|}{$\mathbf{3}$} & \multicolumn{1}{c|}{$\mathbf{4}$} & \multicolumn{1}{c|}{$\mathbf{6}$} & \multicolumn{1}{c|}{$\mathbf{7}$} & \multicolumn{1}{|c|}{$\mathbf{8}$} \\
\hline 1970 & 12.739 & $5,15 \%$ & $94,85 \%$ & 3.768 & $39,73 \%$ & $60,27 \%$ & 16.507 & $82,80 \%$ \\
\hline 1975 & 11.708 & 4,91 & 95,09 & 4.220 & 39,70 & 60,30 & 15.928 & 84,74 \\
\hline 1980 & 9.820 & 1,26 & 98,74 & 5.904 & 39,05 & 60,95 & 15.724 & 86,12 \\
\hline 1985 & 8.083 & 0,73 & 99,27 & 6.721 & 39,28 & 60,72 & 14.804 & 85,85 \\
\hline 1990 & 7.578 & 1,85 & 98,15 & 6.994 & 39,57 & 60,43 & 15.572 & 86,19 \\
\hline 1995 & 6.717 & 0,32 & 99,68 & 7.591 & 46,09 & 53,91 & 14.308 & 85,82 \\
\hline 1996 & 6.717 & 0,35 & 99,65 & 7.715 & 47,91 & 52,09 & 14.432 & 85,11 \\
\hline 2000 & 6.559 & 0,33 & 99,67 & 7.788 & 46,64 & 53,36 & 14.347 & 85,80 \\
\hline 2005 & 6.537 & 0,75 & 99,25 & 8.478 & 49,72 & 50,28 & 15.015 & 85,50 \\
\hline 2009 & 6.394 & 2,13 & 97,87 & 8.936 & 54,22 & 45,78 & 15.330 & 87,11 \\
\hline
\end{tabular}

1: Kilómetros de vía sin electrificar; 2: \% de vía doble; 3: \% de vía única; 4: Kilómetros de vía electrificada; 5: \% de vía doble; 6: \% de vía única; 7: Total de kilómetros de la red ferroviaria; 8: \% de kilómetros pertenecientes a RENFE.

Fuente: Elaboración propia sobre datos del Ministerio de Fomento, 1997. 
de esa notable mejora. Eso no significa que, en un futuro, puedan y deban, buscarse complementariedades y combinaciones adecuadas; si bien éstas no son nada sencillas (Campos et al., 2009). No obstante, la realidad conjunta de las infraestructuras ferroviarias en España es menos brillante que la de los tramos de LAV en funcionamiento refleja. El cuadro 7, detalla unos datos que la resumen.

Los datos de 2009 aún quedan 6.394 Km. de vía sin electrificar, es decir el 41,70\% del total. Así mismo, la vía única abarca $10.347 \mathrm{Km}$., o sea, el 67,49\% del conjunto de la red ferroviaria. Estos dos apartados son definitivos para calibrar la debilidad de nuestras infraestructuras de ferrocarriles. A eso hay que añadir la existencia de extensas áreas en España sin cobertura alguna, por la débil trama de la red ferroviaria nacional y su especial morfología y trazado. Algunas de ellas ya quedaron sin cubrir en sus trazados primigenios; otras son consecuencia de los sucesivos cierres de vías, ante su estado ruinoso y falta de rentabilidad. Su panorama, pues, en conjunto, se caracteriza por rasgos de fuerte debilidad, que apenas admite comparación las redes ferroviarias de otros países vecinos de la Unión, Francia o Alemania. Ya se han hecho algunas breves referencias a las causas que han motivado ese devenir.

Lo expuesto confirma una realidad evidente y contrastada. El sistema ferroviario español se mueve entre una palpable dualidad: una parte significativa de su red ha cambiado poco desde sus trazados iniciales en el siglo XIX. Junto a ella conviven unos trechos recientes, en ascenso, correspondientes a las LAV, que son propios del siglo XXI; están a la vanguardia. Dentro de ese conjunto, el apartado del tráfico de mercancías se encuentra peor y precisa de mayor atención. Hasta ahora no la tiene.

\section{NECESIDAD DE CAMBIO. LA DIFÍCIL MODIFICACIÓN DEL MODELO ACTUAL}

Dada la postración en que se encuentra el transporte de mercancías por ferrocarril, con tendencia incluso a reducirse más, se aconseja actuar sin demora sobre él, mejorándolo. Las motivaciones que sugieren emprender una nueva política de transportes, con mayor equilibrio modal, son de diferente naturaleza, si bien todas convergen en el mismo objetivo. Sólo se hace referencia a las consideradas de mayor significación:

Económicas. De manera objetiva se comprueba que en economías de escala, el coste por unidad de transporte y kilómetro, en distancias medias, al trasladarlas por tierra es más económico el ferrocarril que la carretera (Wackermann, 1995). Esto es más fehaciente en ámbitos, nacionales e incluso internacionales (caso de buena parte de la Unión Europea), siempre que las distancias a cubrir, las superen y no encuentren fácil alternativa en los fletes marítimos (Merlin, 1991, Comisión Europea, 1997). En la medida en que continúe la actual situación española, con la utilización masiva de la carretera, los costes suplementarios incorporados a las mercancías son negativos, a veces casi disuasorios, en un mercando cada vez más abierto y competitivo. Basta considerar el efecto multiplicador que introducen los diferentes elementos implícitos en el coste de los transportes y el precio final de los productos (Julienne, 2002).

Al mismo tiempo, mantener una red ferroviaria con un uso mínimo y a la baja (en el apartado de mercancías), no deja de ser un despilfarro y un derroche económico. A pesar de la ruptura formal del monopolio tradicional, a instancias de la política ferroviaria de la 
Unión (Comisión Europea, (2002), y de la introducción de una tímida liberalización (2005), la reducción de beneficios lastra el resultado final. Todo aconseja cambiar esa dinámica, buscando una situación más equilibrada en la asignación de los menguados recursos públicos (Grahan, 2005).

Ecológicas. El auge que ha tenido en España el transporte por carretera conlleva un mayor consumo energético. Se calcula que representa cerca del $40 \%$ de la demanda final (frente al 34\% de la industria y al $26 \%$ dedicado a otros usos). Ese valor medio supera en casi diez puntos a la magnitud estándar en la Unión Europea (Dron, 1995). Hasta ahora, los combustibles empleados mayoritariamente en el sector del transporte por carretera son derivados del petróleo. Además de la dependencia absoluta que ello significa (dada la carencia de esas fuentes energéticas en España), importa valorar su destacada contribución a la contaminación (Comisión Europea, 2010). Resulta difícil entender la abultada atención que ocupa todo lo relacionado con el uso y empleo de las energías renovables (con una contribución reducida al consumo energético global), mientras que este modo de transporte consume proporciones mucho más elevadas. De ahí las recomendaciones de la Unión Europea en ese sentido (Comisión Europea, 2009). A todas luces es insostenible seguir priorizando el transporte de decenas de millones de toneladas de mercancías, por medio de millones de vehículos. Todo eso introduce una espiral de aumento sin fin, con consecuencias negativas de diferente naturaleza e impacto espacial acusado.

A su vez, el aumento de los flujos de tráfico, y de manera aún mayor el correspondiente a los vehículos pesados, termina colapsando la circulación. La densa red de VRGC construidas en el curso de los últimos lustros, pronto muestra sus limitaciones (Krakovitch, 1996). En numerosos trechos ha sido preciso añadir recientemente nuevos carriles para aumentar su capacidad de circulación y mantener la fluidez de los desplazamientos y dando pie a numerosos accidentes con dolorosos y costosos perjuicios de toda índole. El modelo de transportes de mercancías no puede seguir sustentándose en esa estructura. El consumo de suelo que implica es muy grande. Además, las mayores dificultades se plantean en torno a las aglomeraciones urbanas más extensas y pobladas. Introducir en ellas nuevos tramos de carreteras y autovías para salvar los estrangulamientos surgidos, conlleva arduas obras con costes muy elevados. Algo imposible de atender y mantener a medio y largo plazo. Así mismo, el número de siniestros y pérdida de vidas es alto.

La contaminación acústica es otro apartado que no debe dejarse de lado. La envergadura y la difusión espacial existente en la trama de carreteras por donde se desplazan millones de vehículos trasladando mercancías, contribuye de manera sobresaliente al aumento de decibelios; esto alcanza mayor dimensión en gran parte de las áreas pobladas y más dinámicas de España. Aunque se dediquen elevados presupuestos para aislar y mitigar tales efectos, los resultados difícilmente son satisfactorios para la población residente en sus áreas próximas; un aspecto a considerar, por cuanto añade de negativo.

Estratégicas. El objetivo final de las consideraciones estratégicas, mediante la racionalización, la optimización de usos y recursos y los ahorros oportunos, es alcanzar una ordenación general de los transportes de mercancías (Troin, 1995). Las motivaciones indicadas, de carácter económico y ecológico fundamentalmente, así lo aconsejan. En periodos de evo- 
lución económica positiva ello es aconsejable. Pero, aún resulta más necesario abordarlo en épocas de crisis, cuando se intenta reducir costes. Pero, sobre todo, se precisa un enfoque con visión de largo alcance. Al tratarse de una realidad de naturaleza estructural y amplio calado se necesitan respuestas acordes con los retos planteados. Desde hace años las autoridades de la Unión Europea dedican atención constante a ello (Rus, 2006). Algunas breves referencias se pueden añadir al respecto:

Es prioritaria la elección y puesta en servicio de unos corredores básicos por donde circulen las mercancías. Deben seleccionarse mediante una adecuada combinación de escalas, locales, regionales, nacionales y comunitarias, que cubran y vertebren las necesidades interiores y exteriores de cada entidad territorial (Baboux \& Charrier, 1994). Desde la propia Comisión Europea son numerosos los estudios al respecto (1995 y 2005). Para conseguir esos objetivos el ferrocarril ofrece numerosas respuestas. De ahí las recomendaciones de los órganos pertinentes de la Unión (Comisión Europea, 2008).

Tales corredores deben cumplir un doble objetivo (Tilière \& Hulten, 2003). De una parte, han de servir para el servicio interno, nacional. Así se podrán conectar las principales áreas productoras de bienes y mercancías con las de destino, consumidoras de los mismos, y las áreas de exportación. Debe lograrse una combinación escalar plural y ambiciosa. De otra, es prioritario atender sus necesidades externas. Conviene recordar que la Unión Europea es el principal mercado de consumo mundial, a la vez que la apertura de su economía hacia el exterior precisa dar salida a ingentes cantidades de mercancías (Biehl, 1991). La globalización creciente de los intercambios en el planeta confirma un incremento de estos volúmenes de tráfico de mercancías. La mejora de su accesibilidad adquiere una prioridad básica para proseguir con su desarrollo económico (Vickermann et al. 1999). Si bien las grandes distancias son servidas por el tráfico marítimo, el ferrocarril es quien mejor garantiza la combinación intermodal con las grandes plataformas logísticas y portuarias, tanto para la importación como para la exportación (Ortúzar et al., 2008). Dentro de ese amplio escenario debe insertarse todo planeamiento de organización de los transportes en España (Rus, 2006).

Lo antes apuntado precisa diseñar una armoniosa combinación de escalas entre la demanda y la oferta de bienes, a la cual debe prestar su servicio el mercado de los transportes, por medio de una acorde red de infraestructuras viarias que permita funcionar con comodidad y facilidad, integrando el sistema de relaciones que los transportes de mercancías ofrecen (Seguí \& Martínez, 2004).

La puesta en servicio y el desarrollo complementario de plataformas logísticas, combinadas de manera adecuada con esos ejes básicos de circulación de transportes, constituye también una necesidad perentoria y básica para el transporte racional de mercancías (Auphan, 1997). En este apartado, las carencias españolas son marcadas. Poco se ha hecho; lo realizado, con escaso o nulo planeamiento y, por consiguiente, sin apenas coordinación (Morales, 2010). La geolocalización es clave, al combinar la telemática (tecnología de la información y la comunicación), con la logística; para conseguir una fácil y cómoda distribución de mercancías.

El retroceso continuo de la cuota de mercado del ferrocarril, dentro del transporte de mercancías terrestres es una realidad palpable. Ya se han hecho algunas referencias a ello. Los datos concretos de su reducción, que confirman la gravedad de los hechos, se especifican en el cuadro 8. 
Cuadro 8

HACIA EL OCASO DEL TRANSPORTE DE MERCANCÍAS POR FERROCARRIL EN ESPAÑA. CUOTA SOBRE EL TRANSPORTE TERRESTRE

\begin{tabular}{|l|l|l|l|}
\hline 1993 & 7,80 & 2002 & 5,90 \\
\hline 1994 & 8,05 & 2003 & 5,70 \\
\hline 1995 & 9,70 & 2004 & 4,90 \\
\hline 1996 & 9,80 & 2005 & 4,60 \\
\hline 1997 & 10,30 & 2006 & 4,40 \\
\hline 1998 & 8,30 & 2007 & 4,10 \\
\hline 1999 & 7,90 & 2008 & 4,08 \\
\hline 2000 & 7,30 & 2009 & 4,01 \\
\hline 2001 & 6,80 & 2010 & 3,94 \\
\hline
\end{tabular}

Fuente. Eurostat, 2011.

Los valores más cercanos confirman una situación extrema. Apenas queda margen para seguir esperando. La debilidad actual apremia la toma de decisiones encaminadas a su recuperación.

Por su parte, la estrategia conjunta de la organización de los transportes por parte de la Unión Europea, según se ha apuntado, cada vez plantea con mayor vehemencia la necesidad de su reorganización territorial, a través de unos ejes básicos (Comisión Europea, 2007); a la vez que insta a mejorar su sostenibilidad ambiental. Su consideración como servicio público también contribuye a darle mayor significación (Fournier, 1993). Dentro de ese contexto el Ministerio de Fomento presentó en Madrid (29 de septiembre de 2009) su intención de ocuparse del asunto. Tras un año de estudios (el 14 de septiembre de 2010), ha realizado el denominado «Plan de impulso al transporte ferroviario de mercancías. Los objetivos del Plan son los siguientes:

- Promover la intermodalidad y la colaboración entre modos.

- Mejorar la sostenibilidad medioambiental del sistema de transporte.

- Promover la cooperación entre Administraciones Públicas, especialmente a través de convenios específicos de la Administración General del Estado con las Comunidades Autónomas.

- Impulsar la liberalización del mercado e integrar nuevos actores en el desarrollo de las cadenas logísticas.

- Definir una red eficiente e integrada para el transporte ferroviario de mercancías, resaltando el papel de los no asociados a la misma y la conexión con las redes europeas.

- Dar certidumbre al mercado sobre la definición de la Red y sus condiciones de operación.

- Innovar tanto en sistemas de gestión e información como en procesos operativos.

- Incrementar la calidad y fiabilidad del servicio. 
Tras esos objetivos básicos, su finalidad mediata es incrementar su cuota de mercado. Como referencias se citan Italia (10,4\%), Gran Bretaña (12\%), Francia (16 \%) o Alemania (22\%). La pretensión es ambiciosa; todas superan bastante la cuota de mercado española, multiplicando varias veces la actual.

El horizonte de aplicación de este Plan es 2020, año en que finaliza el PEIT. El Plan engloba 10 grandes programas, 44 acciones y 100 medidas. La mejora de las infraestructuras ferroviarias anunciadas se evalúan en 7.512 M€. Esta suma se desglosa, en esencia, en una inversión de $2.880 \mathrm{M} €$, correspondiente al programa de Infraestructuras Lineales (36 $\%$ del montante de infraestructuras) y $2.520 \mathrm{M} €$ para terminales logísticas. Además, fija la cantidad de $1.800 \mathrm{M} €$ para mejorar la conexión con los puertos y $312 \mathrm{M} €$ para renovar la red FEVE.

Dado que las sumas económicas señaladas son valores elevados, se detalla en el mismo la procedencia de los recursos a utilizar. Así, 5.154 M€ (el 68,6\%) será de inversión pública y $2.358 \mathrm{M} €$ de participación privada $(31,4 \%)$. Se indica que el Ministerio de Fomento aportará la mayor partida mediante la vía de los Presupuestos estatales $\left(4.354 \mathrm{M}^{\mathrm{a}}\right)$; aunque se prevé también la participación de las Autoridades Portuarias (mediante las inversiones tendentes a mejorar las infraestrucuras de acceso a los puertos). Se buscará también la colaboración de las Comunidades Autónomas (si bien no se especifica ni en qué medida, ni a través de que procedimientos o partidas se realizará tal aportación. Se hace, igualmente, referencia al empleo procedente de los fondos europeos, (Marco Polo I. E.) y al programa marco de investigación e innovación. Se espera obtener una partida adicional de $800 \mathrm{M} €$ mediante la venta de terrenos que actualmente son terminales de mercancías, ubicados en áreas centrales de numerosas ciudades.

Además, se buscará la participación privada, centrada en las terminales y accesos a infraestructuras nodales (incluyendo accesos a puertos). Ésta se complementará mediante sociedades mixtas para la promoción y gestión de terminales logísticas. La cantidad que se espera obtener a su través se eleva a $1.008 \mathrm{M} €$. Por último, mediante la vía de las fórmulas de participación pública y público-privadas se estima que puede obtenerse otra cantidad significativa (1.350 M€).

Es posible convenir que la propuesta económica es bien intencionada. Pero, con la situación compleja y difícil que atraviesa el conjunto de la economía nacional, no parece ser el marco adecuado para que buena parte de tales propuestas terminen siendo exitosas. Por hacer sólo alguna mención al respecto, basta pensar en la dificultad de venta en el mercado, según los valores estimados, de semejantes y destacadas reservas de terrenos. También, la propia capacidad de los presupuestos públicos para destinar los fondos señalados a citado apartado, resulta dudosa. En tal caso, se corre el riesgo de que todo este plan sólo sea uno más de los numerosos elaborados y propuestos y, que a la postre, nunca terminaron siendo una realidad, sino meras declaraciones de buenas intenciones (Izquierdo \& Menéndez, 1987).

Ahora bien, esas razonables dudas en cuanto a la financiación y disponibilidad económica, no deben ser un inconveniente central para analizar la necesidad del cambio de signo en la estructura, organización y funcionamiento del actual modelo del transporte de mercancías por ferrocarril. En ese sentido, se aportan unas breves referencias concernientes a aquellos apartados básicos que deben transformase, y sobre los que es conveniente actuar, para evitar la acentuación del declive continuado de este modo de transporte y, en lo posible, revitalizarlo. 
i) Selección de ejes prioritarios. Esto debe emprenderse en aras de conseguir un doble objetivo económico y estratégico. Constituye una prioridad absoluta. Hay que contar con que, dado el conjunto de elementos que conforman la realidad presente, los montantes económicos disponibles, sean de una u otra procedencia, van a ser siempre escasos (La Caixa, 2007). Por ello es necesario priorizar los destinos de las inversiones a realizar. Así mismo, desde una perspectiva estratégica, interesa determinar cuáles son los trechos que cumplen un cometido de mayor recorrido, relacionando áreas prioritarias, bien sean de producción, consumo o tránsito.

Se trata, pues, de seleccionar qué recorridos pueden cumplir el objetivo de transportar mayor volumen de mercancías, en especial de aquellas que es más adecuado encauzarlas por este modo de transporte. Esa acción debe realizarse mediante la combinación equilibrada entre la oferta y la demanda.

El marco espacial de análisis y selección de esos tramos seleccionados debe seguir la escala que abarque la mayor envergadura espacial posible. Se sugiere que contemple tres escenarios de actuación: el internacional, en su sentido más amplio; el exterior, concerniente a los países vecinos, con límites físicos con España, y el marco nacional, en su ámbito interior.

Por lo común, cualquier desviación que se produzca sobre ese horizonte, motivada por consideraciones regionales y territoriales españolas internas, difícilmente tendrá justificación adecuada. Al contrario, será un contratiempo. A nadie se oculta que ello es fácil de decir, también de comprenderlo; aunque resulte difícil seguir esa pauta. Cada vez resulta más evidente el tremendo peso y la consiguiente capacidad de influir en las decisiones políticas estatales procedente de las entidades regionales autonómicas; todas anteponen sus intereses a los comunes nacionales. Pero, igualmente, cada día es más palpable la evidencia de que no hay lugar para particularismos, ni políticas de campanario. La competitividad creciente derivada de un mercado más globalizado, al paso del tiempo, no deja apenas margen de actuación para otras desviaciones auspiciadas por absurdos localismos (Comisión Europea, 2003). La falta de coordinación coherente y sostenible puede conducir hacia un modelo próximo a la balcanización.

La selección de esos ejes, en consonancia con las escalas territoriales señaladas, se deben orientar, como se ha apuntado a cubrir los siguientes objetivos:

a) Por su posición geoestratégica peninsular, la península Ibérica, aunque con cierto desenclave en referencia al área central europea, le cabe cierta posibilidad de desempeñar un modesto papel estratégico como área de intermediación en el copioso tráfico de mercancías que, por barco, surcan los mares y océanos del Planeta. Por ello, es conveniente escoger, seleccionar y adecuar unos puntos de contacto básico, para combinar el transporte intermodal, marítimo-ferroviario de mercancías. Se trata de conjugar un amplísimo foreland con un hinterland nacional y, en su caso, supranacional español; todo ello dentro de su integración en la red europea de transportes (Gerondeau, 1996). Algeciras, Valencia, La Coruña, Gijón, Barcelona y Bilbao, por ejemplo, se presentan como los puntos que, tal vez, pueden reunir mejores ventajas de partida para configurar plataformas logísticas más adecuadas. Las expectativas, en este sentido, existen; si bien no hay que engañarse al comprobar que no están exentas de numerosas dificultades, además de sufrir una intensa competencia desde otros puertos vecinos. 
b) En la medida en que se alcance a cubrir ese objetivo más ambicioso, ello se debe combinar con el propio movimiento de mercancías específico que se genera y relaciona desde y hacia España en su proyección exterior; es decir, el que tenga como origen o destino más significativo los dos países vecinos peninsulares, Portugal y Francia; únicos con los que existe frontera terrestre. Los ejes seleccionados deben orientarse hacia esa estrategia concreta. Sin duda ello no está exento de dificultades, pues no es sencillo combinar nuestros intereses nacionales con los de los vecinos. Es de esperar que ambos procuren abarcar desde sus nodos estratégicos la misma finalidad hacia España. La realidad confirma que casi todo resta por hacer en ese sentido, dadas las limitaciones de infraestructuras y operatividad hoy existente entre nuestra red ferroviaria de mercancías y la de ellos.

c) Las características propias nacionales actuales constituyen el otro ámbito de escala a tener presente. Aquí debe buscarse la difícil armonización entre la realidad existente y su probable evolución futura. Es lógico pensar, atendiendo a su potencial de oferta y demanda, que aquellas áreas en las que se concentran los mayores efectivos humanos y productivos, deben priorizarse a la hora de la selección final. Ésta, como ya se ha apuntado, debe contemplarse con una perspectiva nacional, alejada de los meros intereses regionales y locales. Pero, el actual modelo de infraestructuras radial no se presenta muy oportuno.

ii) Configuración de una red básica de tramos operativos del transporte ferroviario de mercancías para alcanzar una vertebración interior. Esto constituye también un objetivo prioritario. De poco vale contar con unos ejes estructurantes, orientados dentro de un marco territorial más amplio, si no es posible mantener entre sí unas relaciones básicas que posibiliten intercambios fluidos, rápidos y cómodos de mercancías dentro del ámbito nacional. El progresivo incremento de las LAV, con su ancho europeo, aunque ofrezcan potenciales ventajas, a corto plazo, significa así mismo una dificultad añadida. No es fácil armonizarlas con la red convencional. También, debe reiterarse, que todo el trazado de infraestructuras de LAV se ha hecho pensando de inmediato en el tráfico de pasajeros. Para adaptarse al tráfico de mercancías se precisarán numerosas intervenciones con significativos costes. El acondicionamiento de las infraestructuras para los traslados de largos convoy, es, entre otros apartados, uno no desdeñable.

La disposición, básicamente radial, de la red ferroviaria convencional, está siendo, en buena medida, reproducida en la construcción de los nuevos tramos de LAV. Dicha morfología no siempre favorece la configuración de una red básica y operativa que articule gran parte del territorio nacional. Si se desea conseguir eso las inversiones en infraestructuras serán mayores de las previstas y anunciadas. El realismo aconseja contemplar las auténticas posibilidades de actuación presente dentro de una mesura y horizonte más modesto.

Tampoco deben olvidarse las dificultades que acompañan cualquier proceso de diseño de una red básica. Por su propia naturaleza, eso implica selección de objetivos, con el consiguiente descarte de otros. Los intereses regionales, en ciertos casos sustentados en una lógica productiva real, presionarán para imponerse a los nacionales. Una realidad muy compleja se abre ante las actuaciones a realizar en ese sentido. 
iii) Logística y transporte ferroviario de mercancías. La complejidad de elementos intervinientes en el funcionamiento del sistema de transportes, en este caso, de mercancías, sólo se puede abordar de forma adecuada mediante una planificación integral indicativa (Chesnais, 1997). Dentro del amplio apartado de las infraestructuras, un aspecto de singular relevancia es, como ya se ha apuntado, la logística, entendida en su sentido genérico. La idea y el comportamiento tradicional de las estaciones ferroviarias como puntos de engarce singulares de la red de transportes hace tiempo que quedó sobrepasada (Rodrigue et al., 2006). Tampoco resulta útil la vinculación singular y aislada a la red de grandes centros productores o consumidores de mercancías. Entre otras cosas, ello no es válido en España, por la escasa significación de grandes empresas que puedan acogerse a ese esquema de funcionamiento. De ahí la oportunidad que aportan las entidades logísticas, en sus diferentes tamaños, volumen y especialización (polígonos logísticos, plataformas estratégicas y zonas de actuación logísticas, etc.) como espacios de concentración, capaces de organizar la distribución sistemática de los productos. No es preciso insistir en las ventajas, de dispar naturaleza, que pueden conseguirse a través de su instalación y funcionamiento regular. Siempre, su adecuada articulación, se traduce en reducción de costes, gestión más adecuada de los tráficos y beneficios operativos y económicos. Se alcanza esto empleando el tiempo ajustado en los procesos imprescindibles de ruptura de carga, almacenamiento y «stokage» que siempre acompañan los procesos de distribución.

Hasta el día de la fecha, en España no se ha llevado a cabo un programa global de diseño, menos aún de actuaciones, que abarque al conjunto nacional. Se precisa incorporar una nueva concepción de las citadas terminales intermodales como centros logísticos, debidamente jerarquizados, capaces de generar valor añadido. Es cierto que el PEIT (2004), en varios de sus apartados sectoriales, hace mención explícita a la «estructuración y combinación con los diferentes nodos logísticos». Incluso en algunas de sus aportaciones cartográficas se contemplan sus referencias y ubicaciones concretas (véase la figura 1). Pero estas ni siquiera son siempre coincidentes.

Ahora bien, es fácil entender que el diseño de un plan nacional de centros logísticos, jerarquizados, según categorías y con sus diferentes roles a desempeñar, tampoco es algo sencillo de realizar. Las dificultades provienen, tanto de la parte técnica de su análisis, diseño y realización, de los montantes económicos a invertir y de la vertiente política implícita en las decisiones a tomar.

En cuanto a lo primero se trata de realizar un análisis profundo, exhaustivo, donde se incluyan las actividades productivas más necesitadas a causa de su mayor movimiento y volumen de transporte de mercancías, a través de los diferentes modos, en su doble sentido, de oferta y demanda. La dimensión espacial debe ser un apartado esencial en ese proceso de análisis. También aquí, la combinación de las diferentes escalas es imprescindible. Como se apuntó antes, debe abarcarse las tres escalas operastivas indicadas: internacional, nacional y la regional.

Para sufragar los costosos gastos de su inversión se precisa la obligada concurrencia de la inversión privada. No sólo porque ello no sea asumible por los endeudados presupuestos públicos; también porque así podrá buscarse una orientación en la que su agilidad de funcionamiento anuncie mejoras operativas, cobertura de costes e incluso, promesa de ganancias (Arthur Andersen, 1997). 


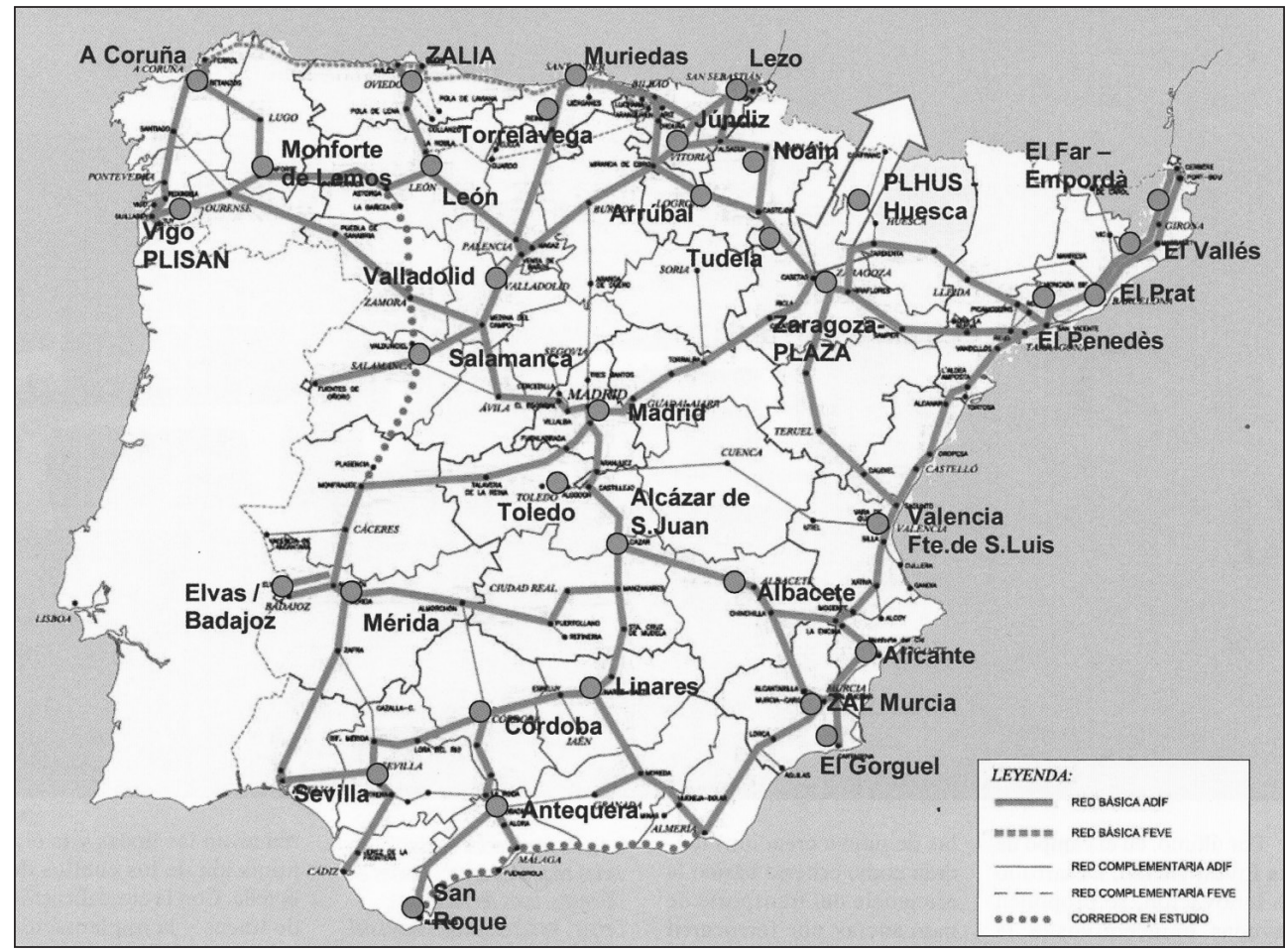

Fuente: Ministerio de Fomento, 2010.

Muy complejo se presenta lo relacionado con las decisiones concretas de selección y jerarquía de entidades logísticas. El sistema descentralizado español, sobre todo atendiendo a la práctica que sigue, beneficia la unilateralidad que lleva a la fragmentación, en lugar de optar por actuaciones globales de interés nacional. No se trata de una afirmación genérica, ni gratuita. Ante la carencia de actuaciones decisivas y coordinadas desde los organismos competentes centrales del Estado, en diferentes autonomías, se están realizando ya ciertas inversiones en determinados nodos seleccionados por ellas, y que, por consiguiente, les favorecen en particular. Su selección se basa en intereses regionales, a veces incluso subregionales o comarcales. Es la política de hechos consumados. Escogida determinada ciudad (por lo común, la capitalidad autonómica) y realizadas las inversiones iniciales pertinentes, a menudo con la ayuda o el aval estatal, para obtener fondos de diversa procedencia, se sientan las bases para aducir y presionar después, señalando que, ese nodo logístico, (aunque sea embrionario), ya debe ser priorizado, en cualquier desarrollo global de la política nacional de transportes. El proceso seguido es el opuesto al que aconseja una acción estatal de interés general, con visión territorial completa. 
iv) La intermodalidad necesaria. Uno de los objetivos esenciales de configurar las adecuadas plataformas logísticas es favorecer la intermodalidad de los transportes de mercancías. El modo marítimo desempeña y, acaso lo hará más en el futuro, un papel destacado en los crecientes volúmenes de mercancías desplazados. El papel que pueden ejercer ciertas plataformas logísticas españolas ubicadas en su litoral, se ha apuntado, que constituye un activo geoestratégico a desarrollar. Incluso, dentro del mero marco territorial europeo, las denominadas autopistas del mar, constituyen una forma de relanzarlo (Comisión Europea, 2010). Los costes reducidos derivados de su utilización representan un acicate para mejorar la competitividad implacable que domina los mercados. La conexión interna entre esas plataformas logísticas de ubicación litoral encuentra en el ferrocarril un modo adecuado para su relación e integración. Más operativa de la que puede ofrecer la carretera (Wackerman, 1995). Todo ello no implica, ni mucho menos su abandono. Este modo de transporte siempre tendrá un campo de actuación destacado, entre otras razones, por su mayor permeabilidad territorial y, en su caso, su menor ruptura de carga. La carretera, dadas las ventajas intrínsecas que aporta, conservará un peso sobresaliente en el transporte de mercancías, en especial en ciertos segmentos y escalas territoriales. De ahí la necesidad de contar con ella en la configuración de una intermodalidad conjunta de los transportes de mercancías.

Pero sí hay que ser conscientes de que el desarrollo del modo de transporte ferroviario, conlleva una reordenación y, a su vez, reconversión del modo de transporte terrestre por carretera. Este asunto representa una dificultad considerable que, sin duda, podrá ser fuente de nuevos problemas. Eso se deduce al calibrar el elevado protagonismo desempeñado por esta última; su abultado volumen empresarial y su consecuente peso laboral Pero, todo ello no debe ser óbice para emprender el camino necesario de potenciar el ferrocarril como modo adecuado de transporte de mercancías

\section{CONSIDERACIONES FINALES}

La realidad que ofrece el transporte de mercancías por ferrocarril en España es débil y frágil; incluso barrunta un colapso definitivo si no se interviene a tiempo. Casi todo está por hacer. Urgen cambios inmediatos pero con perspectivas a medio y largo plazo. La actividad económica española se resentirá con fuerza y perderá más competitividad si no es capaz de conseguir que este modo de transporte alcance mayor significación. Además de esa dimensión económica directa, hay otros apartados indirectos e inducidos que también precisan atención. Sus consecuencias se extienden a ciertos ámbitos colaterales de no menor significación. Me refiero, por ejemplo, a su dimensión ecológica, en aras de aminorar y reducir su contaminación ambiental, acústica y costes derivados de accidentes.

Ahora bien, hay que ser conscientes de las graves dificultades que representa producir un cambio como el precisado. Una realidad tan grave y acentuada exige soluciones ambiciosas de considerable envergadura. Hay que partir de la confección de un planeamiento general de los transportes de ámbito nacional, en el cual, este modo encuentre un protagonismo mayor. La articulación político-territorial de España, y el derrotero seguido en años recientes en su organización regional, no se presume como un escenario favorable para abordar y hacer efectivo ese proceder. Pero, es el camino a recorrer, el que se aconseja seguir. Lo contrario, continuar de forma parcial y con planteamiento fragmentarios, puede traer ciertos éxitos 
puntuales para alguna entidad territorial concreta; la cual, gracias a su capacidad de influir, obtenga ciertas ventajas iniciales. Pero, a la larga, ese proceder se volverá en contra de todas. Al mismo tiempo, es difícil combinar la realidad del mercado transversal con su morfología radial predominante.

No se puede ignorar la creciente interrelación de nuestro mundo globalizado. La respuesta propicia sólo puede venir desde una concepción de los transportes de naturaleza geoestratégica, que abarque el conjunto español e ibérico, incardinado en el ámbito de la Unión Europea y con la mirada puesta en el conjunto mundial. Este planeamiento global de los transportes no es una premisa arbitraria ni caprichosa. Constituye un planteamiento necesario desde donde abordar aspectos intrínsecos imprescindibles. No hay que olvidar que se trata de modificar el actual sistema de transportes existente, sustentado en la carretera, dando mayor protagonismo al ferrocarril. La envergadura del cambio viene marcada también por lo que supone el desarrollo de la intermodalidad como manera básica de actuación. Al mismo tiempo, la puesta en servicio de su logística correspondiente representa una necesidad perentoria, si bien conllevará igualmente una enorme dificultad alcanzarla con éxito.

La dimensión económica que todo ello implica es enorme, las inversiones a realizar cuantiosas. Es tanto lo que hay que hacer que la abultada suma señalada en el plan ministerial presentado puede quedar ampliamente sobrepasada. Esto introduce otro elemento fundamental de preocupación: ¿de dónde obtener la financiación suficiente para su realización? Las propuestas efectuadas no parecen realistas. El panorama actual, de acusada crisis económica, no es el mejor para hacer frente a todo ello. Tampoco lo es el excesivo protagonismo orientado hacia la alta velocidad ferroviaria de pasajeros; un programa de actuaciones deslumbrado con horizontes soñadores, casi quiméricos, con dudosa rentabilidad operativa.

El marco oficial liberalizador del sector ferroviario permite la participación de la iniciativa privada. Interesa estimularla. Otra cosa bien diferente es que las inversiones privadas encuentren motivación e incentivos suficientes para su participación. Eso requiere planteamientos abiertos y liberales que prioricen demandas productivas reales (señaladas hace mucho tiempo ya por A. Smith) en justa combinación con los intereses nacionales.

Son, pues, numerosos los frentes abiertos. Si todos no encuentran la adecuada respuesta este plan oficial presentado, corre el riesgo de quedar sólo en una mera declaración de intenciones. Todo seguiría, en ese caso igual de mal, incluso a peor. Es mucho lo que se juega en ello el devenir de nuestro sistema productivo, pero los interrogantes abiertos son de tal envergadura que transmiten cierta zozobra.

\section{BIBLIOGRAFÍA}

ARTHUR ANDERSEN (1997): Financiación privada de las infraestructuras públicas. Madrid. Ed. Arthur Andersen.

AUPHAN, E. (1997): «Le transport ferroviaire». Les transports. París. Armand Colin, 88-116.

BAUCHET, P. (1996): Les transports en Europe. La trop lente integration. París. Economica.

BAVOUX, J.J. y CHARRIER, J.B. (1994): Transport et structuration de l'espace dans l’Union Européenne. París. Masson. 
BEL, G. (2011): España, capital París. Origen y apoteosis del Estado radial: del Madrid sede cortesana a la «capital total. Barcelona, Destino.

BIEHL, D. (1991): The role of infrastructure in regional development. Infrastructure and Regional Development. Vickermann, R. (ed.): European Research in Regional Science, vol 1,9-35.

CAMPOS MÉNDEZ, J.; RUS MENDOZA, G. y BARRÓN DE ANGOITI, I. (2009): El transporte ferroviario de Alta Velocidad. Una visión económica, Madrid, Fundación BBVA.

COMISIÓN EUROPEA (2002): Libro Blanco. La política europea de transportes de cara al 2010: la hora de la verdad. Luxemburgo.

COMISIÓN EUROPEA (2003): Revitalización de los ferrocarriles europeos. Espacio ferroviario europeo integrado. Bruselas. Dirección General de Energía y Transportes.

COMISIÓN EUROPEA (2005): Trans-European Transport Network, Bruselas. Directorate General for Energy and Transport.

COMISIÓN EUROPEA (2007): Extension of major trans-European Transport axes to the neighbouring countries, Bruselas. Directorate General for Energy and Transport.

COMISIÓN EUROPEA (2008): Un ferrocarril moderno para una Europa moderna. Bruselas. Dirección General de Energía y Transportes.

COMISIÓN EUROPEA (2009): Un futuro sostenible para los transportes. Bruselas. Dirección General de Energía y Transportes.

COMISIÓN EUROPEA (2010): High-speed Europe. A sustainable link Betwenn citizens. Bruselas, Directorate General for Mobility and Transport.

CHESNAIS, M. (1997): «Le transport, élement d'une stratégie complexe ». Les transports. París. Armand Colin, 7-35.

DRON, M. (1995) : Pour une politique soutenable des transports. París. Dossier du Ministere de l'Environnement.

FAITH, N. (1990): The World the railways made. Londres. The Bodley Head.

FERNÁNDEZ DURÁN, R. (2006): El tsunami urbanizador español y mundial. Barcelona, Ed. Virus.

FLYVGJERG, B.M., SKAMRIS, K. y BUHL, S.L. (2004): What Causes cost Overrum in Transport Infrastructure Projects? Transport Review, 24 (1), p. 3-18.

FOURNIER, J. (1993): Le train, l'Europe et le service public. París, Odile Jacobs.

GARCÍA MARTÍNEZ, E. (1987): La política de obras y la política de transportes en España (Una sucinta visión histórica). Situación, $\mathrm{n}^{\circ}$ 1, Banco de Bilbao, 35-46.

GERONDEAU, Ch. (1996): Les transports en Europe. París. EDS Editeur.

GONZÁLEZ VALLVÉ, J. L. y BENEDICTO SOLSONA, M.A. (2006) : La mayor operación de solidaridad de la Historia. Crónica de la Política Regional de la Unión Europea en España. Comisión Europea. Luxemburgo.

GRAHAM, D.J. (2005): Wider Economic Benefits of Transports Improvements: Link Between Agglomeration and Productivity, Londres, DFT, Imperial College.

GUTIÉRREZ PUEBLA, J.; MONZON, A., PIÑERO, J. M. (1992): Accesibilidad a los centros de actividad económica en España. Madrid. Dirección General de Planificación Interregional de Grandes Infraestructuras. M. O. P y Transportes. 
GUTIÉRREZ PUEBLA, J., GÓMEZ, C., GARCÍA PALOMARES, J.C. y LÓPEZ, E. (2006): «Análisis de los efectos de las infraestructuras de transporte sobre la accesibilidad y la cohesión regional», Estudios de Construcción y Transporte, $\mathrm{n}^{\circ} 105$, 215-240.

IZQUIERDO, R. y MENÉNDEZ, J.M. (1987): «Transporte, economía nacional y desarrollo regional», Situación, n 1 . Banco Bilbao-Vizcaya, 5-22.

IZQUIERDO DE BARTOLOMÉ, R. (1992): «La política de transportes en la CE en relación con el sistema ferroviario». I Seminario sobre el ferrocarril. Palma de Mallorca, 27-36.

IZQUIERDO, R. (1993): «Breve evolución histórica del ferrocarril en España: la aparición de la alta velocidad». Situación, Banco Bilbao-Vizcaya, nº 3-4, 7-19.

JULIENNE, Ch. (2002): Le rail \& la route. Pour une nouvelle économie des transports. Paris, Les Belles Lettes.

KRAKOVITCH, A. (1996) : Prévision de la saturation des infrastructures routières en Europe aprés l'an 2000. Estrasburgo. Rapport, Union Européenne.

LA CAIXA (2007): Las infraestructuras. Informe mensual. Servicio de Estudios, Barcelona, mayo.

LÓPEZ PITA, A. (1987): El transporte de viajeros y mercancías por ferrocarril. Situación, 1987, Banco de Bilbao, $n^{\circ}$ 1, 114-131.

MERENNE, E. (1995) : Géographie des transports. París. Nathan, Paris.

MERLIN, P. (1991): Géographie, économie et planification des transports, Paris, P.U.F.

MINISTERIO DE FOMENTO (2004): Plan Estratégico de Infraestructuras y Transportes. Madrid, Secretaría de Estado de Infraestructuras y Transportes.

MOPT (1993): Plan Director de Infraestructuras (1993-2007). Madrid. Secretaría General Técnica, $2^{\text {a }}$ ed. (edición amplia); (edición resumida 38 p).

MORALES GIL, A. (2010): «Las superficies logísticas y la organización espacial de redes de transporte de mercancías en España». Papeles de Geografía, no ${ }^{\circ}$ 51-52, 211-222.

NIJKAMP, P. y VAN GREENHUIZEN, M. (1997): «European transport: Challenges and opportunities for future research and policies». Journal of Transports Geography, vol. 5, 1, 4-11.

ORTUZAR, J.D. y WILLUNSEN, L. (2008): Modelos de transporte. Madrid, Universidad de Cantabria.

QUINET, E. (1992): Infrastructures de transport et croissance. París. Economica.

RODRIGUEZ, J.P., COMTOIS, C. Y SLACK, B. (2006): The Geography of Transport Systems. Londres, Routledge.

RUS MENDOZA, G. de, (Dir.) (2006): La política de transporte europea. El papel del análisis económico. Madrid. Fundación BBVA.

SEGUÍ PONS, J.M. y MARTÍNEZ REYNÉS, M.R. (2004): Geografía de los transportes. Palma de Mallorca, Universidad de las Islas Baleares.

SERRANO MARTÍNEZ, J.M. (2008): «Erreichbarkeit der Städtischen Agglomerationen Spaniens im strassennetz. Modelle und Engpässe». Wurzburger Geographische Manuskripte. Heft/vol. 76, 33-48, Verkehrsgeographische Fallstudien in Europa und seinen Regionen, F. Harder \& K. Schliephake (Eds.).

SERRANO MARTÍNEZ, J.M. y GARCÍA MARÍN, R. (2010): «La política de transporte ferroviario en España. Los corredores de Alta Velocidad: sus potenciales y limitaciones». 
Scripta Nova. Revista Electrónica de Geografía y Ciencias Sociales. Vol. XIV, núm. 331 (20), 1 de agosto de 2010.

TILIÈRE, G. De, y HULTEN, S. (2003): «A decade of change in the European Rais market; Influence on Innovation and R\&D: Toward a new equilibrium in the railway sectorEPRC». Toulouse. Transport Conference Papers, 234-248.

TROIN, J. F. (1995) : Rail et aménagement du territoire, des héritages aux nouveaux défis. París. Edisud.

VICKERMANN, R.; SPIEKERMANN, K. y WEGENER, M. (1999): «Accesibility and Economic Developpement in Europe». Regional Studies Association, vol. 33, 1, 1-15.

VV.AA. (2009): Panorama of transport. Eurostat, Luxemburgo.

WACKERMANN, G. (1995): Le transport de marchandises dans l'Europe de demain. Le défi de l'équilibre. Paris, La Recherche, Midi Éditeur.

WAIS, F. (1974): Historia de los ferrocarriles en España. Madrid. Editora Nacional. 